\title{
A Wideband Digital Frequency Synthesizer
}

\author{
Yi-Chuan Liu, Chung-Cheng Wang, Terng-Yin Hsu, Chen-Yi Lee \\ Dept. of Electronics Engineering, National Chiao Tung University \\ 1001, University Road, Hsinchu 300, Taiwan, ROC \\ E-mail: ycliu@royals.ee.nctu.edu.tw
}

\begin{abstract}
With the rapid advance in CMOS technology, the trend of the VLSI then towards system-on-chip (SOC) where design methodology, cost, and turnaround time are major issues. Concepts of intellectual property (IP) are then proposed to fit for SOC designs. Based on a DFS controller IP [4]. a wideband digital frequency synthesizer (DFS) is proposed to fit in with the wireless LAN applications, which provides low cost and efficient design periods. A 2-stage voltage-controlled oscillator (VCO) is designed to achieve the system requirements and generates such a high-speed frequency. Its output frequency range can be from $465 \mathrm{MHz}$ to $2.635 \mathrm{GHz}$ with double $3.0-\mathrm{V}$ supplies. Besides, a novel cell-based digital-to-voltage converter (DVC) is also proposed to solve the interface between the DFS controller IP and the VCO. The wideband DFS is fabricated in 0.35-um SPQM CMOS technology.
\end{abstract}

\section{INTRODUCTION}

In Wireless LAN transceiver design, we need a high-speed oscillator to produce a target frequency in the $900 \mathrm{MHz}$ and $2.4 \mathrm{GHz}$ ISM bands. In order to generate the target frequency in a stable manner, a frequency synthesizer is usually used to create such a high-speed frequency from a low-speed input reference clock. There are many architectures proposed for these applications [1][2][3]. but most of them are analog designs.

The TDC-based DFS architecture. like all digital phase-locked loop architecture [4], can also be applied to these kinds of applications in high integration. Because the controlling parts of the DFS can be used as an intellectual property, a digital frequency synthesizer can be designed in a low cost and high efficiency. We only need to design a digital-controlled oscillator (DCO), with frequency ranges falling on the $900 \mathrm{MHz}$ and $2.4 \mathrm{GHz}$ bands.

Though a cell-based DCO is always preferred. it has some fundamental limits. A major problem is that such a high frequency band cannot be achieved by a cell-based DCO. Another problem is its high power consumption at high frequency. This is because a cell library is often designed for general purpose and an ASIC seldom works at so high frequency. It may achieve those specifications by careful design. but extreme cost will be paid indeed

To overcome those problems, a DCO with full custom design is required. A DCO architecture proposed in [6] provides a possible solution. A voltage-controlled oscillator can also be used but some additional circuits are needed to handle the interface between the DFS controller and the VCO. In order to obtain better qualities of the output clock, a VCO is preferred in this design and a circuit called digital-to-voltage converter (DVC) is developed to deal with the interface issue. If we combine the DVC and the VCO together, it is also a DCO indeed. Block diagram of this frequency synthesizer is shown in Figure 1.

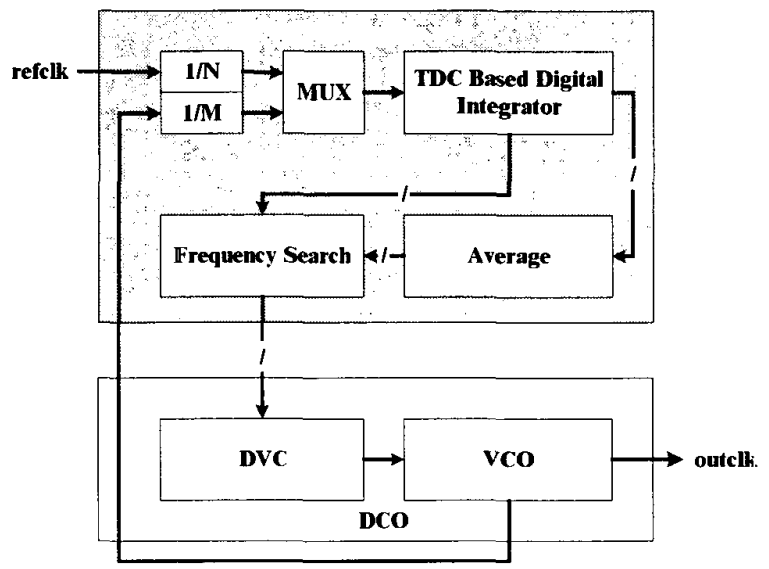

Figure 1. Block Diagram of the Wideband Digital Frequency Synthesizer, which is based on a DFS controller IP.

To explain how the new DCO is obtained. some issues about designing the proposed DCO are dealt with in Section 2. Section 3 presents the detail circuit of the proposed cell-based DVC and 2-stage differential delay cell techniques. In Section 4. simulation results are described and followed by concluding remarks in Section 5 .

\section{DESIGN OF PROPOSED DCO}

\subsection{DVC Basic Concepts}

The controlling signals in the DFS controller are all digital but the VCO accepts only voltage, which is an analog signal. Then an interface is needed to handle this conversion. Since the resolution of the converted voltage influences the frequency step of the VCO, which is related to the stability and accuracy of the whole DFS, a DAC with high resolution is also required. According to the concept of the voltage transfer curve (VTC) of a static CMOS inverter, as shown in Figure 2.. a novel circuit named digital-to-voltage converter (DVC) is developed.

A CMOS inverter acts not only an inverter itself but also a voltage-to-voltage converter. If the inputs are restricted only to 
the states of high and low, it just performs the inverter function. But if all voltage levels are allowed in the input, it performs the voltage-to-voltage conversion. That is, one input voltage maps into one output voltage. If we are able to quantize voltage levels at the input, then a digital-to-voltage converter is obtained in a simple way.

Quantization of voltage levels at the input can be achieved by the circuit, which is illustrated in Figure 3. This structure is similar to the parallel connection of inverters except all inverter inputs are not tied together. If each inverter input is connected together, it can just be regarded as a super inverter with large driving capability. The separate inputs are the key point to perform the function of digital-to-voltage conversion.

The size of each inverter can be equal, binary-weighted or whatever configurations. All the input, $\operatorname{In}[0] \sim \operatorname{In}[n-1]$, are digital signals. That is, they can only be at the state of either high or low. According to the functionality of an inverter, an input at low state will pull up the output to logic high. On the other hand, an input at high state will pull down the output to logic low. Since each input can be either high or low and each output is tied to the same node, the output will be at some voltage level between the high and low according to the input configurations. All inputs at low state will let the output at high and all inputs at high state will let the output at low. The more the inputs are at high. the lower the output voltage level. Some simulation results of the simple DVC structure are shown in Figure 4. There are 256 inverters with equal size in these simulations.

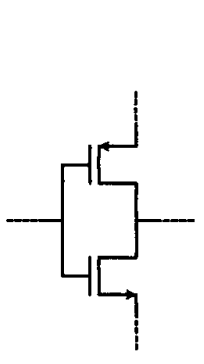

(a)

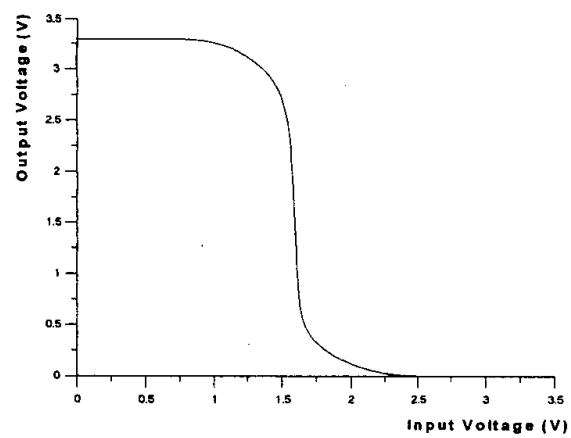

(b)
Figure 2. (a) Schematic of static CMOS inverter, and (b) its transfer curve.

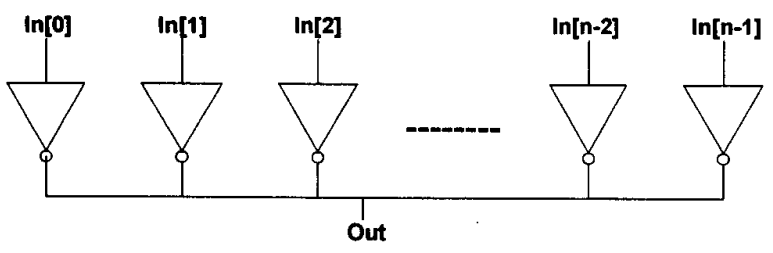

Figure 3. A simple DVC architecture.

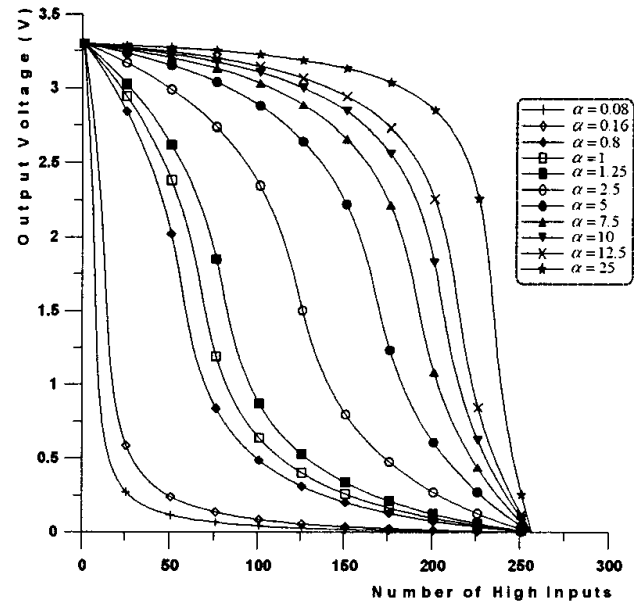

Figure 4. Simulation results of the simple DVC structure with 256 equal-size inverters. $\alpha=\mathrm{wp} / \mathrm{wn}$; the channel width ratio of the PMOS and NMOS.

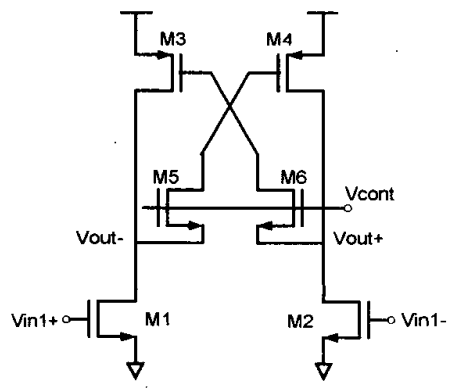

Figure 5. Differential Delay Cell.

\subsection{Differential Delay Cell}

The circuit of the proposed delay cell is shown in Figure 5. This cell has a differential structure to reduce the power-supplyinjected phase noise [7]. A tail current source MOS transistor. which is commonly used in a conventional emitter-coupled logic (ECL)-like differential CMOS pair, is avoided to reduce 1/f noise. A pair of PMOS load transistors. M3 and M4. are added to the delay cell to constitute a CMOS latch. The cross-coupled NMOS transistors. M5 and M6, control the maximum gate voltage of the PMOS load transistors and limit the strength of the added latch. When $V_{\text {cont }}$ is slow, the strength of the latch becomes weak, and the output driving current of the PMOS load increases. Therefore. the state of the latch is changed easily. and the delay time is reduced. Thus, when $V_{\text {cont }}$ is high, the latch becomes strong, and it resists the voltage switching in the differential delay cell. As a result, the delay time increases. With the help of the positive feedback of the latch, the transition edges of the output waveform remain sharp in spite of slow delay time. Since the delay cell is basically a simple differential inverter. full-swing waveform is generated from the ring oscillator. These facts make the delay cell perform complete switching and reduce the portion of ontime in the oscillaton period. 


\section{CIRCUIT DESCRIPTION}

\subsection{Advanced DVC Structure}

The major drawback of the simple DVC is its non-linearity, and it has to be modified to fit in our application. The output characteristic is approximately linear when small variations around the operating point are applied to the input. Thus. input words of the DVC are divided into two parts. One part is for the coarse tune and the other is for the fine tune. The coarse-tune process is similar to determine the operating point of the DVC and the fine-tune process is then to make small changes in the input. Since the operating point is determined. the relation between the output voltage and the fine-tune words is almost linear.

To achieve the operations described above, we use tri-state inverters instead of the ordinary ones and make something difference at each input node. There are two types of tri-state inverters. The one with normal driving capability is used for the coarse tune and the one with very small driving capability is used for the fine tune. We reserve one inverter for pulling up the output voltage to the voltage high. The input pin of each tri-state inverter is always connected to the voltage high. Oppositely, the input of the individual inverter is always connected to the voltage low. The enable pin of each tri-state inverter now becomes the digital input of the DVC. The structure of the modified DVC is shown in Figure 6(a). Besides, there are two groups of input words in this DVC structure. One is called "Coarse" which is used for the coarse tune and the other is called "Fine" which is used for the fine tune. The controlling scheme of this DVC is shown in Figure 6(b)

Because the input of the individual inverter is always connected to ground. the voltage level at the output node will be at voltage high if all the controlling words. Coarse and Fine, are off. When one or more of the controlling words are on, the voltage level at the output node will be pulled down toward to the ground. The more controlling words are on. the lower the output voltage is. The output voltage reaches its lowest value, which is determined by the characteristic of the inverter and the tri-state inverters. when all the controlling words, including Coarse and Fine, are on Since every tri-state inverter in each part is the same. the output voltage is not determined by the turned-on order but is determined by the turned-on number. With the modified structure and the controlling scheme. we can greatly improve the linearity of the DVC.

\subsection{2-Stage, and High-Speed Ring Oscillator}

In a conventional ring oscillator, the oscillation frequency is determined as $1 /(2 \mathrm{~N} \tau)$, where $\mathrm{N}$ is the number of stages and $\tau$ is the unit delay time of a delay cell. Hence the frequency of the oscillator is decided by the delay time of one delay element. The delay time cannot be smaller than that of a single inverter: therefore, the maximum frequency of the VCO is linited by the delay time of the basic inverter delay cell. To solve this frequency-limitation problem. a single-end ring oscillator with a negative skewed delay scheme has been reported [8]. The proposed delay cell is single ended, and the oscillator is sensitive to the power-supply noise and not controllable.
In this work, by using a dual-delay [7] and 2-stages [9] concepts to implement the VCO, higher operation frequency and wider turning range are achieved simultaneously. The modified $\mathrm{VCO}$ is shown in Figure 7. The differential delay cell is modified to combine dual-delay path into single delay path. A pair of PMOS transistors, M2 and M5, are added to the delay cell to compensate the PMOS performance and prematurely turn on the PMOS during the output transition. This operation enhances the unit delay time, $\tau$, of the output and contributes to reducing the phase noise of the overall VCO. Moreover, using 2-stage differential delay cell can also reduce the number of stages, $\mathrm{N}$, and solve the frequency-limitation problem.

For this modified differential delay cell, the higher controlled voltage, $\mathrm{V}_{\text {cont }}$, the larger current NMOS transistors pass and hence the faster the output frequency.

\section{SIMULATION RESULTS}

The post-layout simulations of the proposed design are performed in 3.0-V, and 0.35-um CMOS SPQM technology. Because there are too many control states in the DCO, only two curves are shown in figure8. The horizontal axis indicates the DCO coarse-tune stages and the curves shows the voltage changes with various coarse-tune words fixed at certain fine-tune control states. The lower curve is fixed at the stage where all of fine-tune tri-state inverters are turned on. Oppositely, the upper curve is fixed at the stage where all of fine-tune tri-state inverters are turned off. Between these two curves. there are 256 curves indicating the other fine-tune stages and are not illustrated for clearness. Minimum voltage resolution that the advanced DVC can achieve is about $0.25 \mathrm{mV}$.

Figure 9 shows the tuning characteristic of $\mathrm{VCO}$. The horizontal axis indicates the controlled voltage and curve shows the frequency changes with various voltages. Figure 10 shows the $1.8 \mathrm{GHz}$ output waveform of VCO. The peak-to-peak voltage is about $2-\mathrm{V}$ with a $1.5-\mathrm{V}$ DC offset.

The whole system can be simulated under Verilog with the aid of the DCO behavior model. This helps us to verify the function and timing of the whole synthesizer in a short term before migrating to the physical design. In order to simulate the influence of the input jitter and verify our canceling scheme, a reference clock model with $20 \%$ jitter is introduced under Verilog simulation. The Verilog simulation result is illustrated in Figure 11.

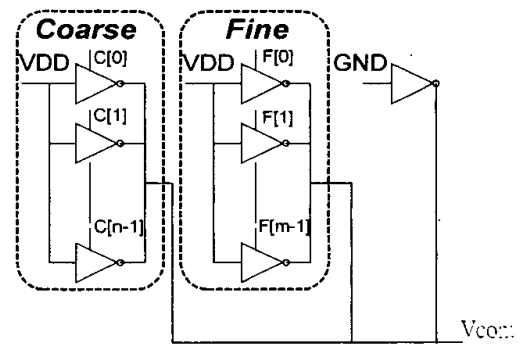

(a)

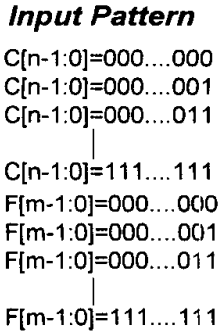

(b)
Figure 6. (a) Modified DVC structure. and (b) its input pattern from DFS controller. 


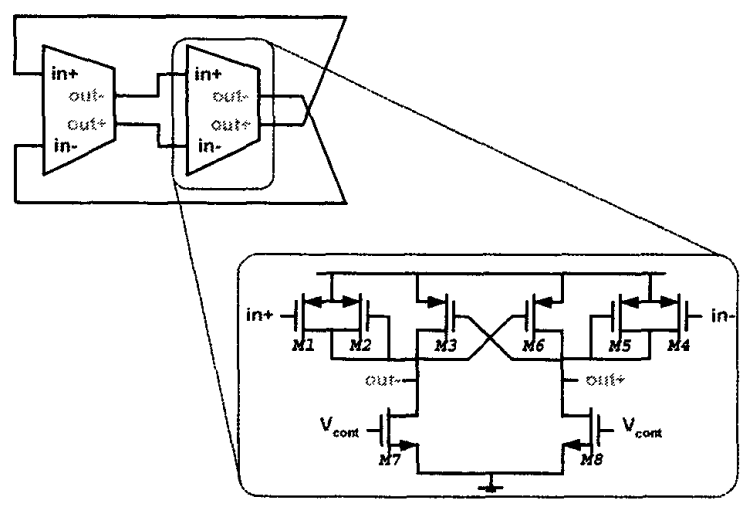

Figure 7. Modified 2-stage VCO structure.

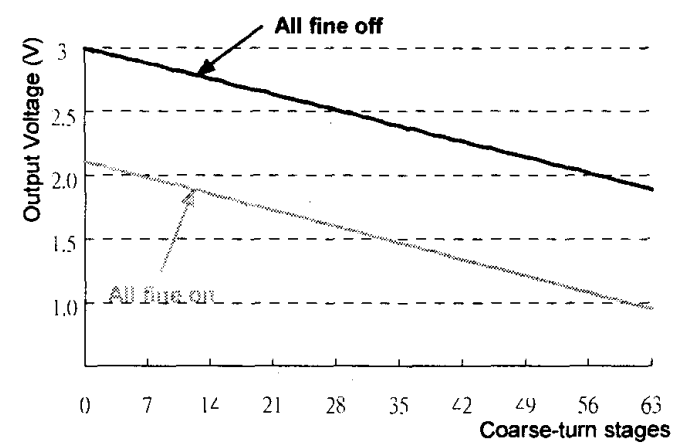

Figure 8. DVC tuning characteristics.

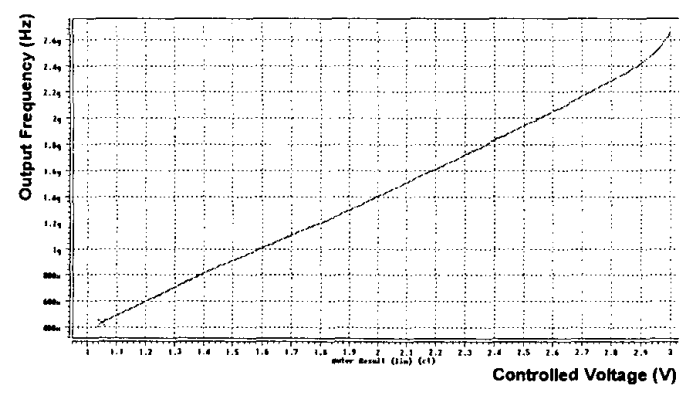

Figure 9. VCO tuning characteristics.

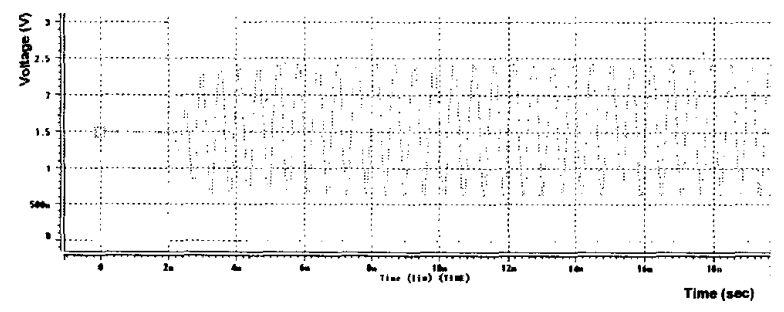

Figure 10. VCO Output Waveform@1.8GHz.

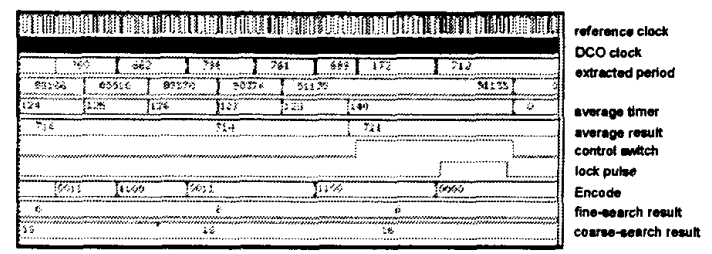

Figure 11. System simulation of proposed design.

\section{SUMMARY}

Based on this DFS controller IP, a wideband digital frequency synthesizer is proposed to fit in with the wireless LAN applications. A full-custom voltage-controlled oscillator (VCO) is designed to achieve the system requirements and generate such a high-speed frequency. In order to solve the interface between the DFS controller IP and the VCO and provide a digital-tovoltage conversion, a novel cell-based digital-to-voltage converter (DVC) is proposed, too. This DVC only uses inverters and tri-state inverters. which are common and various elements in a cell library. The cell-based manner gives a low-cost and portable design methodology of the digital-to-voltage conversion.

The post-layout simulation shows the DFS has the frequency range between $465 \mathrm{MHz}$ and $2.635 \mathrm{GHz}$ with resolution about of $13 \mathrm{KHz}$. In addition. the total power dissipation is under $80 \mathrm{~mW} @ 1.8 \mathrm{GHz}$.

\section{REFERENCES}

[1] J. Craninckx. M. S. J. Steyaert. "A Fully Integrated CMOS DCS-1800. Frequency Synthesizer," IEEE J. Sold-State Circuits, vol. 33, no. 12, pp. 2054 2065. Dec. 1998.

[2] S. J. Lee, B. Kim, K. Lee, "A Fully Integrated Low-Noise 1$\mathrm{GHz}$ Frequency Synthesizer Design for Mobile Communication Application," IEEE J. Sold-State Circuits, vol. 32, no. 5, pp. 760 765. May 1997.

[3] M. Thamsirianunt, T. A. Kwasniewski, "A 1.2mm CMOS Implementation of a Low-Power $900-\mathrm{MHz}$ Mobile radio Frequency Synthesizer," IEEE Custom Integrated Circuits Conference 1994, pp. 383 386.

[4] Terng-Yin Hsu. Bai-Jue Shieh. Chen-Yi Lee, "An alldigital phase-locked loop (ADPLL)-based clock recovery circuit," IEEE J. Solid-State Circuits, vol. 34, no. 8, pp.1063 -1073.Aug.1999.

[5] J. Dunning. G. Garcia. J. Lundberg, E. Nuckolls, "An AllDigital Phase-Locked Loop with 50-Cycle Lock Time Suitable for High-Performance Microprocessors." IEEE $J$. Sold-State Circuits. vol. 30, no. 4. pp. 412 422. April 1995.

[6] Chan-Hong Park. Beomsup Kim. " A Low-Noise, 900MHz VCO in 0.6um CMOS," IEEE J. Solid-State Circuits, vol. 34. no. 5. pp. 586-591, May 1999.

[7] S.-J. Lee, B. Kim. and K. Lee. "A novel high-speed ring oscillator for multiphase clock generation using negative skewed delay scheme." IEEE J. Solid-State Circuits, vol.32. pp. 289-291, Feb. 1997.

[8] Yeon Kug Moon; Kwang Sub Yoon, "A 3.3V high speed CMOS PLL with a two-stage self-feedback ring oscillator." AP-ASIC '99, pp. $287-290,1999$. 